\title{
Risk of Incident Dementia and Cognitive Impairment in Patients with Chronic Obstructive Pulmonary Disease (COPD): A Large UK Population-based Study
}

RA Siraj ${ }^{1,2}$, TM McKeever ${ }^{1,2}$, JE Gibson ${ }^{2}$, AL Gordon $^{3,4}$, CE Bolton ${ }^{1}$

\section{Authors affiliations:}

${ }_{1}^{1}$ NIHR Nottingham Biomedical Research Centre Respiratory Medicine, School of Medicine, University of Nottingham, City Hospital NUH Trust site, Nottingham, UK

${ }^{2}$ Division of Epidemiology and Public Health, School of Medicine, University of Nottingham, Nottingham, UK

${ }^{3}$ Division of Medical Sciences and Graduate Entry Medicine, School of Medicine, University of Nottingham, Derby, UK

${ }^{4}$ NIHR Nottingham Biomedical Research Centre, Musculoskeletal Theme, School of Medicine, University of Nottingham, Nottingham, UK

\section{Correspondence to:}

Professor C.E. Bolton,

Respiratory Medicine,

NIHR Nottingham Biomedical Research Centre Respiratory Medicine,

School of Medicine, University of Nottingham,

City Hospital Campus,

Hucknall Road, Nottingham,

NG5 1PB. UK

Tel: 01158231054

Email: charlotte.bolton@nottingham.ac.uk

Keywords: COPD, Cognitive impairment, Dementia 


\section{Abstract}

Background: Although cognitive impairment and dementia are common comorbidities in patients with chronic obstructive pulmonary disease (COPD), estimates of incidence following a diagnosis of COPD are inconclusive.

Objective: To determine the incidence of cognitive impairment and dementia in people with and without a COPD diagnosis.

Methods: A population-based study using UK General Practice (GP) health records from The Health Improvement Network database was conducted. Patients with confirmed COPD diagnosis, $\geq 40$ years old, were matched to up to four subjects without a COPD diagnosis by age, sex and GP practice. Cox proportional hazards models were used to assess the incidence rates of cognitive impairment and dementia.

Results: Of patients with COPD ( $n=62,148), 9 \%$ developed cognitive impairment, compared with $7 \%$ of subjects without COPD $(n=230,076), p<0.001$. The incidence of cognitive impairment following COPD diagnosis was greater than in subjects without COPD following index date (adjusted Hazard Ratio (aHR), 1.21; 95\% Cl: $1.16-1.26, \mathrm{p}<0.001$ ). The coded incidence of either cognitive impairment or dementia was also greater in patients with COPD following adjustment for confounders (aHR: $1.13,95 \% \mathrm{Cl}: 1.09-1.18, \mathrm{p}<0.001$ ). Coded incident dementia alone was not different between patients with COPD and subjects without COPD (aHR, 0.91, 95\% Cl: $0.83-1.01, p=0.053)$.

Conclusion: Despite the increased incidence of cognitive impairment in patients with COPD, incident of dementia was not as frequently recorded in patients with COPD. This raises the concern of undiagnosed dementia and emphasises the need for a systematic assessment in this population. 


\section{Introduction}

Cognitive impairment is a common comorbidity in Chronic Obstructive Pulmonary Disease (COPD), contributing to greater functional disability $(1,2)$, increased need for care services (3), lower adherence to complex medication regimens (4), worse clinical outcomes (5) and predicting mortality in certain COPD populations (6).

The reported prevalence of cognitive impairment in COPD is estimated between $4 \%$ to $61 \%$ $(7,8)$, depending on the study populations, assessment methods, and measures of COPD severity $(9,10)$. Current evidence suggests that patients with COPD are at increased risk of cognitive impairment and subsequent dementia. However, the existing studies investigating the incidence of cognitive impairment and dementia in COPD are limited by methodological issues, such as study design, population age, inadequate matching group, and short followup (11-14), meaning that the estimated incidence is caveated.

Potential mechanisms contributing to impaired cognitive state in patients with COPD are likely multiple and include ageing, co-existent cardiovascular (CV) disease, smoking, hypoxemia and hypercapnia (15). Recent evidence suggests that patients with COPD and cognitive impairment are at increased risk of developing dementia (11). Identifying the proportion of patients with COPD at increased risk of cognitive impairment and dementia is important in understanding the broader clinical spectrum of COPD and in providing services that are structured to meet patient needs.

This study aimed to assess the incidence of newly diagnosed cognitive impairment and dementia following a diagnosis of COPD and compare this to subjects without COPD using primary care electronic health records within the UK. 


\section{Method}

\section{Data source and study population}

The study population was obtained using The Health Improvement Network (THIN), a UK database containing anonymised patient records from over 550 general practices (GPs) (16). The index population comprised individuals aged $\geq 40$ years with a new Read-coded COPD diagnosis from $1^{\text {st }}$ April 2006 to $31^{\text {st }}$ December 2015, with $\geq 1$ year of record prior to the COPD diagnosis (17). Each patient was matched by age, gender and GP practice with up to four subjects without a diagnosis of COPD, and each subject without COPD was assigned the same index date as the matched patients with COPD. The date at which dementia coding was introduced as part of the UK GP quality and outcomes framework (QOF) was used as the start of the study period (01-04-2006) (18). Ethical approval was obtained from an independent Scientific Review Committee (ref. 17THIN095).

\section{Outcome measures and follow-up}

$\underline{\text { Incident cognitive impairment }}$

Subjects with a code of cognitive impairment and dementia prior to the index date were excluded. The first recorded event of cognitive impairment following the index date was considered. Subjects were followed-up from the index date until either they received a cognitive impairment Read code, left the GP practice, died or reached the end of the followup (31/12/2015), whichever came first.

\section{$\underline{\text { Incident dementia }}$}

A former diagnosis of dementia prior to index date was excluded from analyses related to dementia incidence. Incident dementia was defined as the first READ coded recorded after the index date.

Diagnosis for both cognitive impairment and dementia were defined based on Read codes in consultation with a geriatrician. An inclusive approach was taken where any potentially relevant diagnosis was considered. 


\section{Potential confounders}

Several explanatory variables and potential confounders were extracted including age, gender and Townsend social deprivation score at COPD diagnosis (19). Clinical covariates included smoking status and the Medical Research Council (MRC) dyspnoea scale (20) recorded closest to the index date. A modified Charlson Comorbidity Index (CCI) (21), excluding dementia and CV disease (congestive heart failure, myocardial infarction, and peripheral vascular disease), was also determined before or at index date. Having received $\geq 1$ prescription for inhaled corticosteroids or oral corticosteroids within 1 year of index date (before or after index date) was also considered as a risk factor.

\section{Statistical analyses}

The demographic and clinical characteristics of subjects were described in terms of frequency or mean and standard deviation as appropriate. Categorical data were compared using McNemar's test. Incidence rates were calculated for both groups using Cox proportional hazard regression to estimate the hazard ratio $(\mathrm{HR})$ for the risk of cognitive impairment following COPD diagnosis. Conditional analyses were conducted to account for matching age, sex, and GP surgery. Confounders were included in the final model if they independently changed the HR for cognitive impairment by $\geq 5 \%$. Similar analyses were conducted to yield the HR of dementia.

A sensitivity analysis was conducted amongst patients with COPD using the MRC dyspnoea score. The incidence of coded vascular dementia was determined in patients with COPD and subjects without COPD. STATA 15.0 software (StataCorp LP, College Station, TX, US) was used for data management and statistical analyses. 


\section{Results}

A total of 64,397 patients with COPD and 243,420 subjects without COPD were included in the analyses, representing those without a former code of dementia. The characteristics of subjects included are presented in Table 1. The whole population had a mean (SD) age of 65.8 (10.8) and 53\% were males. The median follow-up period was 4.4 years across the groups. The prevalence of cognitive impairment without a diagnosis of dementia at baseline was greater for patients with COPD $(n=2,182 ; 3.4 \%)$ than for subjects without COPD ( $n=6,869$; $2.9 \%), p<0.001$. Dementia was present in $1.1 \%$ of patients with COPD compared to $1.7 \%$ of subjects without COPD, $\mathrm{p}<0.001$.

\section{Cognitive impairment following index date}

After excluding subjects with former coded cognitive impairment and/or dementia, there were 62,148 patients with COPD and 230,076 subjects without COPD, and demographics remained similar.

Of patients with COPD, 5,545 (9\%) patients had a doctor recorded diagnosis of cognitive impairment, compared to $15,693(7 \%)$ of subjects without COPD, $p<0.001$. The incidence rate of cognitive impairment in patients with COPD was 25.1 per 1,000 person-years $(95 \% \mathrm{Cl}$ : 24.3-25.6) compared with 17.6 per 1,000 person-years (95\% Cl: 17.3-17.9) in subjects without COPD. Patients with COPD were $21 \%$ more likely to have a recorded incidence of cognitive impairment compared with subjects without COPD (adjusted HR [aHR]: 1.21 (95\% $\mathrm{Cl} 1.16$ to 1.26$) ; \mathrm{p}<0.001$; Table 2) following adjustment for confounders.

The association of MRC dyspnoea score amongst patients with COPD at time of COPD diagnosis with cognitive impairment was evaluated. There were 36,621 patients with MRC score 1-3 and 4,677 with MRC score 4-5. There was a significantly greater incidence of cognitive impairment in patients with MRC score of 4-5 compared to 1-3 following adjustment age, sex, socioeconomic class, and smoking. (aHR: $1.10 ; 95 \% \mathrm{Cl}: 1.01$ to $1.20 ; \mathrm{p}=0.040$ ). In the fully adjusted model, the association was attenuated (Table 3). 


\section{Dementia following index date}

Following COPD diagnosis, 1,151 (1.8\%) from the population of 64,397 had a recorded incidence of dementia compared with 4,609 (1.9\%) subjects without COPD ( $n=243,420)$ $p=0.077$. The incidence rate of dementia in patients with COPD and in subjects without COPD was 4.9 per 1,000 person-years. There was no association between patients with COPD and incidence of dementia following adjustment for confounders (aHR: $0.91,95 \% \mathrm{Cl}: 0.83$ to 1.01 ; Table 2).

Similar proportions of subjects in both groups with cognitive impairment had an incident diagnosis of dementia (around 11\%). In patients with COPD, those with diagnosed cognitive impairment were seven times more likely to develop dementia than those without cognitive impairment (aHR: 7.19; 95\% Cl: 6.07-8.53; p<0.001). A similar finding was also observed in subjects without COPD.

Following COPD diagnosis, the risk of vascular dementia was greater in patients with COPD than subjects without COPD matched for age, gender and GP (HR: 1.27; 95\% Cl: 1.13-1.44; Table 2). This association was attenuated following further adjustments (aHR: $1.14 ; 95 \% \mathrm{Cl}$ : $0.94-1.37 ; p=0.160)$.

Patients with COPD with an MRC score of 4-5 $(n=4,949)$ were at greater risk of incident dementia, compared with patients with an MRC score 1-3 ( $n=37,999)$, following adjustment for confounders (aHR: $1.30,95 \% \mathrm{Cl}: 1.05$ to 1.61 ; Table 3 ).

\section{Incidence of either cognitive impairment or dementia}

There were 6,524 (10.50\%) patients with COPD $(n=62,148)$ with a record of either cognitive impairment or dementia, compared with 19,475 (8.50\%) subjects without COPD ( $n=230,076)$, $p<0.001$. Patients with COPD were more likely to have a recorded incidence of either cognitive impairment or dementia compared to subjects without COPD (aHR: 1.13, 95\% Cl: 1.09 to 1.18; Table 2).

The incidence of either cognitive impairment or dementia was greater in patients with COPD with an MRC score of 4-5 compared with patients with an MRC score 1-3, following adjustments for age, sex, socioeconomic class, and smoking (aHR: 1.12, 95\% Cl: 1.02 to 1.22; $\mathrm{p}=0.010$ ). CV disease and modified $\mathrm{CCl}$ diminished the relationship (Table 3). 


\section{Discussion}

The coded incidence of cognitive impairment was significantly greater in patients with COPD compared to subjects without COPD after adjustment for confounders. In contrast, the incidence dementia was not different between patients with COPD and subjects without COPD. To the best of our knowledge, this is the first study to investigate this in patients with COPD using UK electronic GP records.

Cognitive impairment is present in $4 \%$ of older patients ( $\geq 65$ years old) with mild COPD (7) and up to $61 \%$ in patients with COPD and severe hypoxemia (8). This wide variation is likely due to the severity of COPD (10), whether cognitive impairment was systematically assessed or self-reported, and use of different diagnostic criteria and tools for cognitive impairment (9). In addition, studies had different threshold definitions for cognitive impairment, even when using the same cognitive assessment tool. The proportion of coded cognitive impairment at COPD diagnosis in the GP electronic health records presented in this study (3.4\%) was far lower than the proportion in clinical studies reported as $40-50 \%$ when cognitive impairment was systematically assessed $(22,23)$. This raises a concern of subclinical underdiagnosed cognitive impairment, which may lead to a delayed intervention; and consequently, a missed opportunity to reduce the impact of cognitive impairment on this vulnerable population.

Current evidence suggests that patients with COPD are at amplified risk of cognitive impairment and even dementia. Our finding that patients with COPD had a higher incidence of cognitive impairment is consistent with prior literature $(12,24,25)$. A recent study from Finland showed that a diagnosis of COPD in midlife was associated with $85 \%$ increased risk of mild cognitive impairment/dementia in later life (12). However, the latter study incorporated self-reported COPD as opposed to a COPD diagnosis that is objectively confirmed. We also found that patients with COPD and cognitive impairment were at a greater risk of dementia compared with patients with COPD without cognitive impairment. In this context, establishing accurate estimates of the proportion of patients with COPD and cognitive impairment could help to further understand the clinical relevance of cognitive decline in patients with COPD and identify a high-risk group. This is also important to allow for appropriate therapeutic interventions and changes in lifestyle, which could prevent or delay the cognitive deterioration to clinical dementia.

There is a growing evidence linking cognitive impairment with COPD, yet literature on dementia in COPD is lacking, and prior studies are also limited by study population (13), selfreported assessment of COPD (12), short or even undefined follow-up time $(13,26)$, and inadequate matching group (27). Contrary to expectations, we found no association between COPD and incident dementia. Although at first glance these findings suggest that patients with 
COPD may not be at a greater risk of dementia, there is a possibility that a dementia diagnosis in patients with COPD has been unintentionally missed. It is likely that COPD symptoms may predominate and be the central focus during GP visits. In addition, doctors may find limited explanatory value in attaching a label of dementia to cognitive impairment in the context of multi-system functional decline as COPD progresses. It is, however, possible that treatment opportunities and, in many jurisdictions, the opportunity to access dementia-specific services are being missed, ultimately to the detriment of the patient. Therefore, the formalisation of dementia might not be addressed accordingly. These findings reinforce the need for a systemic assessment to the cognitive state of patients with COPD during routine clinical follow up.

The association between COPD severity and cognitive impairment has been highlighted (10). Hung et al. showed that a self-reported diagnosis of severe COPD (defined by oxygen use and limited physical activity) was associated with a rapid cognitive decline (11), highlighting the potential increased risk of subsequent dementia. Using MRC dyspnoea score as a severity measure (20), our study is consistent that those with more breathlessness were at greater risk of cognitive impairment and dementia compared to those with a better MRC score. This finding has potential important clinical implications, as cognitive impairment and dementia interfere with patient's ability to adhere to their medications and manage their own disease $(4,28)$, which may consequently lead to worse health outcomes $(6,7)$.

Patients with COPD usually present with a smoking history and have a wide range of comorbidities, one of which is CV disease (29). As expected, in this study, the proportions of current smoking and CV disease were greater in patients with COPD and these confounders attenuated the association with vascular dementia. Because COPD and CV comorbidities share similar risk factors and patients with COPD are more likely to be predisposed to cardiovascular events (30), it is therefore difficult to determine whether COPD is associated with increased risk of vascular dementia. Although It is unlikely for CV comorbidities or smoking per se to account for the vascular dementia in COPD, it is important to encourage healthcare professionals to identify and manage any modifiable risk factors.

A recent cross-sectional study suggested a different pattern of cognitive impairment, across domains to those seen in Alzheimer's Dementia (31). Establishing whether patients with COPD has a specific pattern of cognitive impairment is indeed of clinical importance, as each type of cognitive impairment may be assessed, managed, and approached differently. 


\section{Strengths and limitations}

A strength of this study is that using electronic primary care health records provides a representative sample for the general UK COPD population (16). The data source includes longitudinal data on various potential risk factors, such as demographic factors and medical conditions. Further, the analyses adjusted for a wide range of potential confounders. Patients with COPD from 2006 onwards were included, after QOF had been already established, meaning that COPD diagnoses were based on spirometry (17). However, there is still a possibility of COPD misdiagnosis (32). In addition, retrospective analyses based on medical records that were mainly collected for administrative purposes rather than research are subject to some limitations. There is a possibility that free text (which is largely inaccessible for research due to issues around patient confidentiality) instead of Read codes may be used to record this information. Although QOF was introduced to improve the quality of recording, some variables used in this study may have changed from the last recorded coded entry, such as self-reported smoking. The possibility of residual confounding of important risk factors, such as hypoxia, cannot be excluded. Information on oxygen saturations and oxygen therapy are not well recorded in READ codes; thus, these factors could not be adjusted for in the analyses.

\section{Conclusion}

In this large population-based study, patients with COPD were associated with increased risk of incident cognitive impairment, whereas no association was observed with dementia. This highlights the potential likelihood of under-diagnosis of dementia with missed opportunity for proper intervention and underlining the need to accurately estimate the proportion of patients with COPD and dementia with a systematic assessment. 
Table 1. Demographic and clinical characteristics for patients with COPD and subjects without COPD without a prior code of dementia, matched by age, gender and GP surgery

\begin{tabular}{|c|c|c|c|c|c|}
\hline \multirow{2}{*}{ Characteristics } & \multicolumn{2}{|c|}{ Patients with COPD } & \multicolumn{2}{|c|}{ Subjects without COPD } & \multirow[b]{2}{*}{$p$-value } \\
\hline & $\mathrm{n}=64,397$ & $\%$ & $\mathrm{n}=243,420$ & $\%$ & \\
\hline Age at diagnosis (years) & $66.4(10.9)$ & & $65.7(11)$ & & \\
\hline $\begin{array}{l}\text { Follow-up } \\
\text { (years, median, IQR) }\end{array}$ & 4.4 & $2.3-6.7$ & 4.4 & $2.3-6.7$ & \\
\hline \multicolumn{6}{|l|}{ Gender } \\
\hline Male & 34,071 & 52.91 & 127,707 & 52.46 & \\
\hline Female & 30,326 & 47.09 & 115,713 & 47.54 & \\
\hline \multicolumn{6}{|l|}{ Townsend score } \\
\hline 1 least deprived & 10,456 & 16.24 & 59,169 & 24.31 & \multirow{5}{*}{$<0.001$} \\
\hline 2 & 11,397 & 17.70 & 54,267 & 22.29 & \\
\hline 3 & 13,032 & 20.24 & 47,880 & 19.67 & \\
\hline 4 & 14,693 & 22.82 & 42,350 & 17.40 & \\
\hline 5 most deprived & 12,363 & 19.20 & 29,684 & 12.19 & \\
\hline Unknown/missing & 2,456 & 3.81 & 10,070 & 4.14 & \\
\hline \multicolumn{6}{|l|}{ MRC dyspnoea score } \\
\hline $1-3$ & 37,999 & 59.01 & 2,619 & 1.08 & \multirow{3}{*}{$<0.001$} \\
\hline $4-5$ & 4,949 & 7.69 & 172 & 0.07 & \\
\hline Missing & 21,449 & 33.31 & 240,629 & 98.85 & \\
\hline \multicolumn{6}{|l|}{ Smoking status } \\
\hline Never smoked & 5,346 & 8.30 & 114,125 & 46.88 & \multirow{3}{*}{$<0.001$} \\
\hline Ex-smoker & 35,008 & 54.36 & 87,705 & 36.03 & \\
\hline Current smoker & 23,971 & 37.22 & 33,928 & 13.94 & \\
\hline Unknown & 72 & 0.11 & 7,662 & 3.15 & \\
\hline \multicolumn{6}{|l|}{ Modified CCl } \\
\hline $0-1$ & 43,959 & 68.26 & 177,949 & 73.10 & \multirow{4}{*}{$<0.001$} \\
\hline 2 & 10,266 & 15.94 & 35,362 & 14.53 & \\
\hline 3 & 5,356 & 8.32 & 16,920 & 6.95 & \\
\hline$\geq 4$ & 4,816 & 7.48 & 13,189 & 5.42 & \\
\hline \multicolumn{6}{|l|}{ Medications } \\
\hline Oral corticosteroids & 27,229 & 42.28 & 15,808 & 6.49 & $<0.001$ \\
\hline \multicolumn{6}{|l|}{ Comorbidities } \\
\hline Diabetes mellitus & 8,395 & 13.04 & 38,505 & 12.33 & $<0.001$ \\
\hline CV disease (CHF, IHD, PVD) & 16,253 & 25.24 & 34,862 & 15.82 & 0.001 \\
\hline Cerebrovascular disease & 5,321 & 8.26 & 13,632 & 5.60 & $<0.001$ \\
\hline
\end{tabular}

Abbreviations: $\mathrm{CCl}$, Charlson comorbidity index; CHF: chronic heart failure; CV: cardiovascular; IHD: Ischemic heart disease; IQR, interquartile range; MRC: Medical Research Council; PVD: peripheral vascular disease 
Table 2. Univariate and multivariate Cox regression models of the incidence of cognitive impairment, dementia alone, either cognitive impairment or dementia, and vascular dementia for patients with COPD compared with subjects without COPD

\begin{tabular}{|c|c|c|c|c|c|}
\hline & $\begin{array}{l}\text { Number of } \\
\text { events }\end{array}$ & $\begin{array}{c}\text { Rate/1000 } \\
\text { person-years }\end{array}$ & $\begin{array}{c}\text { Crude HR } \\
(95 \% \mathrm{Cl})\end{array}$ & $\begin{array}{c}\text { Fully adjusted } \\
\text { HR (95\%Cl) }\end{array}$ & $P$ value \\
\hline \multicolumn{6}{|l|}{ Cognitive impairment } \\
\hline $\begin{array}{c}\text { Subjects without } \\
\text { COPD }\end{array}$ & 15,693 & $\begin{array}{c}17.6 \\
(17.3 \text { to } 17.9)\end{array}$ & 1.00 & 1 & \\
\hline Patients with COPD & 5,545 & $\begin{array}{c}25.1 \\
\text { (24.3 to } 25.6)\end{array}$ & $\begin{array}{c}1.47 \\
(1.43 \text { to } 1.52)\end{array}$ & $\begin{array}{c}1.21 \\
(1.16 \text { to } 1.26)^{a}\end{array}$ & $<0.001$ \\
\hline \multicolumn{6}{|l|}{ Dementia } \\
\hline $\begin{array}{c}\text { Subjects without } \\
\text { COPD }\end{array}$ & 4,609 & $\begin{array}{c}4.9 \\
(4.7 \text { to } 5.1)\end{array}$ & 1 & 1 & \\
\hline Patients with COPD & 1,151 & $\begin{array}{c}4.9 \\
(4.7 \text { to } 5.2)\end{array}$ & $\begin{array}{c}1.01 \\
(0.94 \text { to } 1.07)\end{array}$ & $\begin{array}{c}0.91 \\
(0.83 \text { to } 1.01)^{b}\end{array}$ & 0.053 \\
\hline
\end{tabular}

\section{Cognitive impairment or dementia}

\begin{tabular}{|c|c|c|c|c|c|}
\hline $\begin{array}{c}\text { Subjects without } \\
\text { COPD }\end{array}$ & 19,475 & $\begin{array}{c}22.1 \\
(21.7 \text { to } 22.4)\end{array}$ & 1 & 1 & \\
\hline Patients with COPD & 6,524 & $\begin{array}{c}29.7 \\
\text { (29.1 to } 30.4)\end{array}$ & $\begin{array}{c}1.39 \\
(1.34 \text { to } 1.43)\end{array}$ & $\begin{array}{c}1.13 \\
(1.09 \text { to } 1.18)^{\mathrm{c}}\end{array}$ & $<0.001$ \\
\hline \multicolumn{6}{|l|}{ Vascular Dementia } \\
\hline $\begin{array}{l}\text { Subjects without } \\
\text { COPD }\end{array}$ & 1,396 & $\begin{array}{c}1.4 \\
(1.4 \text { to } 1.5)\end{array}$ & 1 & 1 & \\
\hline Patients with COPD & 426 & $\begin{array}{c}1.8 \\
\text { (1.6 to } 2.1)\end{array}$ & $\begin{array}{c}1.27 \\
(1.13 \text { to } 1.44)\end{array}$ & $\begin{array}{c}1.14 \\
(0.94 \text { to } 1.37)^{d}\end{array}$ & 0.160 \\
\hline \multicolumn{6}{|c|}{$\begin{array}{l}\text { Crude HR: Cox regression models-derived HR was adjusted for age, sex and GP. } \\
\text { Fully adjusted: } \\
\text { a Multivariate Cox regression-derived HR was adjusted for age, sex, GP, smoking status, modified CCI, CV disease, corticosteroid } \\
\text { use, and socioeconomic class. } \\
\text { b Multivariate Cox regression-derived HR was adjusted for age, sex, GP, BMI, smoking status, modified CCI, CV disease, and } \\
\text { socioeconomic class. } \\
{ }^{\circ} \text { Multivariate Cox regression-derived HR was adjusted for age, sex, GP, BMI, smoking status, modified CCI, CV disease, } \\
\text { corticosteroid use, and socioeconomic class } \\
\text { d Multivariate Cox regression-derived HR was adjusted for age, sex, GP, smoking status, modified CCI, CV disease, and } \\
\text { socioeconomic class } \\
\text { Abbreviations: BMI: body mass index; CCl, Charlson comorbidity index; Cl: confidence interval; CV: cardiovascular; GP: general } \\
\text { practice; HR: hazard ratio }\end{array}$} \\
\hline
\end{tabular}


Table 3. Comparison of incidence of cognitive impairment, dementia, and either cognitive impairment or dementia in patients with COPD according to MRC dyspnoea score (1-3 vs. 4-5)

MRC dyspnoea score $\quad$ Adjusted $\mathrm{HR}(95 \% \mathrm{Cl}) \quad$ Fully adjusted $\mathrm{HR}(95 \% \mathrm{Cl}) \quad \mathrm{P}$ value

\section{Cognitive impairment}

$\begin{array}{cccc}\begin{array}{c}1-3 \\ (n=36,621)\end{array} & 1 & 1 & \\ & & & \\ 4-5 & & & \\ (n=4,677) & 1.11(1.02-1.22) & 1.03(0.93-1.13)^{a} & 0.522\end{array}$

\section{Dementia}

$1-3$

$(n=37,999)$

1

1

$4-5$

$(\mathrm{n}=4,949)$

$1.31(1.06-1.61)$

$1.30(1.05-1.61)^{a}$

0.012

Either cognitive impairment or dementia

\begin{tabular}{|c|c|c|c|}
\hline $\begin{array}{c}1-3 \\
(n=36,621)\end{array}$ & 1 & 1 & \\
\hline $\begin{array}{c}4-5 \\
(n=4,677)\end{array}$ & $1.14(1.04-1.24)$ & $1.07(0.98-1.16)^{a}$ & 0.131 \\
\hline \multicolumn{4}{|c|}{$\begin{array}{l}\text { Adjusted HR: Cox regression models-derived HR was adjusted for age and sex. } \\
\text { Fully adjusted: } \\
\text { a Multivariate Cox regression-derived HR was adjusted for age, sex, BMI, smoking status, modified CCI, CV disease, } \\
\text { corticosteroid use, and socioeconomic class. } \\
\text { Abbreviations: BMI: body mass index; CCl: Charlson comorbidity index; CI: confidence interval; CV: cardiovascular; GP: } \\
\text { general practice; HR: hazard ratio; MRC: Medical Research Council. }\end{array}$} \\
\hline
\end{tabular}




\section{References}

1. Hartley P, Gibbins N, Saunders A, Alexander K, Conroy E, Dixon R, et al. The association between cognitive impairment and functional outcome in hospitalised older patients: a systematic review and meta-analysis. Age and ageing. 2017;46(4):559-67.

2. Martinez $\mathrm{CH}$, Richardson $\mathrm{CR}$, Han MK, Cigolle CT. Chronic obstructive pulmonary disease, cognitive impairment, and development of disability: the health and retirement study. Annals of the American Thoracic Society. 2014;11(9):1362-70.

3. Fogg C, Meredith P, Bridges J, Gould GP, Griffiths P. The relationship between cognitive impairment, mortality and discharge characteristics in a large cohort of older adults with unscheduled admissions to an acute hospital: a retrospective observational study. Age and ageing. 2017;46(5):794-801.

4. Allen SC, Jain M, Ragab S, Malik N. Acquisition and short-term retention of inhaler techniques require intact executive function in elderly subjects. Age and ageing. 2003;32(3):299-302. 5. Campbell SE, Seymour DG, Primrose WR. A systematic literature review of factors affecting outcome in older medical patients admitted to hospital. Age and ageing. 2004;33(2):110-5.

6. Antonelli-Incalzi R, Corsonello A, Pedone C, Trojano L, Acanfora D, Spada A, et al. Drawing impairment predicts mortality in severe COPD. Chest. 2006;130(6):1687-94.

7. Chang SS, Chen S, McAvay GJ, Tinetti ME. Effect of coexisting chronic obstructive pulmonary disease and cognitive impairment on health outcomes in older adults. Journal of the American Geriatrics Society. 2012;60(10):1839-46.

8. Grant I, Prigatano GP, Heaton RK, McSweeny AJ, Wright EC, Adams KM. Progressive neuropsychologic impairment and hypoxemia. Relationship in chronic obstructive pulmonary disease. Archives of general psychiatry. 1987;44(11):999-1006.

9. Yohannes AM, Chen W, Moga AM, Leroi I, Connolly MJ. Cognitive Impairment in Chronic Obstructive Pulmonary Disease and Chronic Heart Failure: A Systematic Review and Meta-analysis of Observational Studies. Journal of the American Medical Directors Association. 2017;18(5):451.e1.e11.

10. Schou L, Ostergaard B, Rasmussen LS, Rydahl-Hansen S, Phanareth K. Cognitive dysfunction in patients with chronic obstructive pulmonary disease--a systematic review. Respiratory medicine. 2012;106(8):1071-81.

11. Hung WW, Wisnivesky JP, Siu AL, Ross JS. Cognitive decline among patients with chronic obstructive pulmonary disease. American journal of respiratory and critical care medicine. 2009;180(2):134-7.

12. Rusanen M, Ngandu T, Laatikainen T, Tuomilehto J, Soininen H, Kivipelto M. Chronic obstructive pulmonary disease and asthma and the risk of mild cognitive impairment and dementia: a population based CAIDE study. Current Alzheimer research. 2013;10(5):549-55.

13. Xie F, Xie L. COPD and the risk of mild cognitive impairment and dementia: a cohort study based on the Chinese Longitudinal Health Longevity Survey. International journal of chronic obstructive pulmonary disease. 2019;14:403-8.

14. Thakur N, Blanc PD, Julian L, Yelin EH, Katz PP, Sidney S, et al. COPD and cognitive impairment: the role of hypoxemia and oxygen therapy. International journal of chronic obstructive pulmonary disease. 2010;5:263-9.

15. Dodd JW, Getov SV, Jones PW. Cognitive function in COPD. The European respiratory journal. 2010;35(4):913-22.

16. Blak BT, Thompson M, Dattani H, Bourke A. Generalisability of The Health Improvement Network (THIN) database: demographics, chronic disease prevalence and mortality rates.

Informatics in primary care. 2011;19(4):251-5.

17. Quint JK, Mullerova H, DiSantostefano RL, Forbes H, Eaton S, Hurst JR, et al. Validation of chronic obstructive pulmonary disease recording in the Clinical Practice Research Datalink (CPRDGOLD). BMJ open. 2014;4(7):e005540. 
18. Lester $\mathrm{H}$, Sharp DJ, Hobbs FD, Lakhani $M$. The quality and outcomes framework of the GMS contract: a quiet evolution for 2006. The British journal of general practice : the journal of the Royal College of General Practitioners. 2006;56(525):244-6.

19. Townsend $P$, Phillimore $P$, Beattie $A$. Health and deprivation: inequality and the North: Routledge; 1988.

20. Mahler DA, Ward J, Waterman LA, McCusker C, ZuWallack R, Baird JC. Patient-reported dyspnea in COPD reliability and association with stage of disease. Chest. 2009;136(6):1473-9.

21. Charlson ME, Pompei P, Ales KL, MacKenzie CR. A new method of classifying prognostic comorbidity in longitudinal studies: development and validation. Journal of chronic diseases. 1987;40(5):373-83.

22. Yohannes AM, M NE, Holbrook JT, Sugar EA, Henderson R, Baker AM, et al. Association of mild cognitive impairment and characteristic of COPD and overall health status in a cohort study. Expert review of respiratory medicine. 2021;15(1):153-9.

23. Cleutjens F, Spruit MA, Ponds R, Vanfleteren L, Franssen FME, Gijsen C, et al. Cognitive impairment and clinical characteristics in patients with chronic obstructive pulmonary disease. Chronic respiratory disease. 2018;15(2):91-102.

24. Lutsey PL, Chen N, Mirabelli MC, Lakshminarayan K, Knopman DS, Vossel KA, et al. Impaired Lung Function, Lung Disease, and Risk of Incident Dementia. American journal of respiratory and critical care medicine. 2019;199(11):1385-96.

25. Singh B, Mielke MM, Parsaik AK, Cha RH, Roberts RO, Scanlon PD, et al. A prospective study of chronic obstructive pulmonary disease and the risk for mild cognitive impairment. JAMA neurology. 2014;71(5):581-8.

26. Liao KM, Ho CH, Ko SC, Li CY. Increased Risk of Dementia in Patients With Chronic Obstructive Pulmonary Disease. Medicine. 2015;94(23):e930.

27. Liao WC, Lin CL, Chang SN, Tu CY, Kao CH. The association between chronic obstructive pulmonary disease and dementia: a population-based retrospective cohort study. European journal of neurology. 2015;22(2):334-40.

28. Arlt S, Lindner R, Rosler A, von Renteln-Kruse W. Adherence to medication in patients with dementia: predictors and strategies for improvement. Drugs \& aging. 2008;25(12):1033-47.

29. Dal Negro RW, Bonadiman L, Tognella S, Bricolo FP, Turco P. Extent and prevalence of cognitive dysfunction in chronic obstructive pulmonary disease, chronic non-obstructive bronchitis, and in asymptomatic smokers, compared to normal reference values. International journal of chronic obstructive pulmonary disease. 2014;9:675-83.

30. Mullerova H, Agusti A, Erqou S, Mapel DW. Cardiovascular comorbidity in COPD: systematic literature review. Chest. 2013;144(4):1163-78.

31. Morris C, Mitchell JW, Moorey H, Younan HC, Tadros G, Turner AM. Memory, attention and fluency deficits in COPD may be a specific form of cognitive impairment. ERJ open research. 2019;5(2).

32. Strong M, Green A, Goyder E, Miles G, Lee AC, Basran G, et al. Accuracy of diagnosis and classification of COPD in primary and specialist nurse-led respiratory care in Rotherham, UK: a crosssectional study. Primary care respiratory journal : journal of the General Practice Airways Group. 2014;23(1):67-73. 